\title{
THE $p$-ADIC LEFSCHETZ $(1,1)$ THEOREM IN THE SEMISTABLE CASE, AND THE PICARD NUMBER JUMPING LOCUS
}

\author{
Go YAMASHITA
}

\begin{abstract}
We prove a semistable version of the so-called $p$-adic Lefschetz $(1,1)$ theorem. As an application, we show a generalization of the Maulik-Poonen result on Picard number jumping locus.
\end{abstract}

\section{Introduction.}

Let $K$ be a complete discrete valuation field of mixed characteristic $(0, p)$ whose residue field $k$ is perfect. Let $X$ be a proper semistable model over $S:=\operatorname{Spec} O_{K}$, $X_{K}$ be its generic fiber $X \otimes_{O_{K}} K$, and $Y$ be its special fiber $X \otimes_{O_{K}} k$. For an element $[L] \in \operatorname{Pic}^{\log }(Y):=H^{1}\left(Y,\left(\mathcal{O}_{Y}^{\times}\right)^{\log }\right):=H^{1}\left(Y, M_{Y}^{\mathrm{gp}}\right)$ or $[L] \in \operatorname{Pic}(Y)$, we have a log-crystalline first Chern class

$$
c_{\text {crys }}([L]) \in H_{\text {crys }}^{2}\left(\left(Y, M_{Y}\right) /\left(W, N^{0}\right)\right),
$$

where $W$ is the ring of Witt vectors with coefficient $k$, and $M_{Y}$ and $N^{0}$ are logstructures on $Y$ and Spec $W$ respectively (the precise meaning of notations will be explained later). We also have Hyodo-Kato isomorphism ([HK])

$$
\rho_{\pi}: H_{\text {crys }}^{m}\left(\left(Y, M_{Y}\right) /\left(W, N^{0}\right)\right) \otimes_{W} K \cong H_{\mathrm{dR}}^{m}\left(X_{K} / K\right) .
$$

This isomorphism depends on the choice of a uniformizer $\pi \in K$. However, we can show that $\rho_{\pi}\left(c_{\text {crys }}([L])\right)$ is independent of the choice of $\pi$ (Corollary 2.3). In this paper, we first show the following generalization of the Berthelot-Ogus theorem ([BO, Theorem (3.8)]):

Theorem 0.1. (=Theorem 3.1) The element $[L] \in \operatorname{Pic}^{\log }(Y)_{\mathbb{Q}}\left(\right.$ resp. $\left.[L] \in \operatorname{Pic}(Y)_{\mathbb{Q}}\right)$ lifts to $\operatorname{Pic}^{\log }(X)_{\mathbb{Q}}\left(\right.$ resp. $\left.\operatorname{Pic}(X)_{\mathbb{Q}}\right)$, if and only if

$$
\rho_{\pi}\left(c_{\text {crys }}([L])\right) \in H_{\mathrm{dR}}^{2}\left(X_{K} / K\right)
$$

is in $F^{1} H_{\mathrm{dR}}^{2}\left(X_{K} / K\right)$.

Next, by using this theorem, we deduce a generalization of the Maulik-Poonen result ([MP]) (see Section 4 for precise meaning of the notations):

Received by the editors September 29, 2009. Revision received November 16, 2010.

Supported by EPSRC grant EP/E049109/1. Partially supported by TOYOTA Central R\&D Labs., Inc. 
Theorem 0.2. (=Theorem 4.3) Let $C$ be the completion of an algebraic closure of $K$, and $O_{C}$ be its valuation ring. Let $B$ be an irreducible separated $O_{K}$-scheme of finite type, and let $f: \mathcal{X} \rightarrow B$ be a proper semistable morphism such that $f_{K}: \mathcal{X}_{K} \rightarrow B_{K}$ is smooth (i.e., for any point $b \in B\left(O_{K}\right)$, the pull-back $\mathcal{X}_{b} \rightarrow \operatorname{Spec} O_{K}$ is a semistable model of the proper smooth variety $\left.\mathcal{X}_{b} \otimes_{O_{K}} K\right)$, where ()$_{K}$ 's mean $K \otimes_{O_{K}}$.

Then, the set

$$
B\left(O_{C}\right)_{\text {jumping }}:=\left\{b \in B\left(O_{C}\right) \mid \rho\left(\mathcal{X}_{b} \otimes_{O_{C}} \bar{k}\right)>\rho\left(\mathcal{X}_{\bar{\eta}}\right)\right\}
$$

is nowhere dense in $B\left(O_{C}\right)$ for the analytic topology, where $\rho$ 's mean (log) Picard numbers.

In Section 1, we review Hyodo-Kato isomorphism, introducing some notations. In Section 2, we study log-crystalline first Chern class and de Rham first Chern class. In Section 3, we prove Theorem 0.1. In Section 4, we prove Theorem 0.2 by using Theorem 0.1 .

\section{Notations.}

Let $K$ be a complete discrete valuation field of mixed characteristic $(0, p)$ whose residue field $k$ is perfect. Let $O_{K}$ denote the valuation ring of $K$. Let $W$ be the ring of Witt vectors with coefficient $k$, and $K_{0}$ be its fraction field. Let $\sigma$ denote the Frobenius on $W$ or $K_{0}$. We use the convention that the subscript ()$_{n}$ of rings, schemes, $\log$-structures, etc. means $\otimes \mathbb{Z} / p^{n} \mathbb{Z}$. Here, "log-structure" means the Fontaine-IllusieKato $\log$-structure ([K1]). When we use the word "log-structure" in this paper, then this means fine saturated log-structure.

Let $X$ be a proper semistable model over $S:=\operatorname{Spec} O_{K}, X_{K}$ be its generic fiber $X \otimes_{O_{K}} K$, and $Y$ be its special fiber $X \otimes_{O_{K}} k$. Let $M$ be the log-structure on $X$ defined by the special fiber $Y$, and $M_{Y}$ be the pull-back of $M$ to $Y$. Let $N$ be the $\log$-structure on $S$ defined by its special fiber Spec $k$. Let $N_{1}$ be the pull-back of $N$ to $\operatorname{Spec} k$, and $N_{n}^{0}$ be the log-structure on Spec $W_{n}$ associated to $\Gamma\left(\operatorname{Spec} k, N_{1}\right) \rightarrow k \stackrel{[\cdot]}{\rightarrow} W_{n}$, where $[\cdot]$ is the Teichmüller representative. We have a natural exact closed immersion $\left(\operatorname{Spec} W_{n}, N_{n}^{0}\right) \hookrightarrow\left(\operatorname{Spec} W_{n+1}, N_{n+1}^{0}\right)$.

We will use the PD-structures $\gamma$ on $\left(W_{n}, p W_{n}\right)$ and $\left(S_{n}, p \mathcal{O}_{S, n}\right)$ etc. induced by the unique PD-structure on $(W, p W)$ and $\left(S, p \mathcal{O}_{S}\right)$ etc. We abbreviate the log-crystalline site $\left(\left(Y, M_{Y}\right) /\left(W_{n}, N_{n}^{0}, p W_{n}, \gamma\right)\right)_{\text {crys }}^{\log }$ as $\left(\left(Y, M_{Y}\right) /\left(W_{n}, N_{n}^{0}\right)\right)_{\text {crys }}^{\log }$ etc. If we abbreviate the coefficient of crystalline cohomology, then it means that the coefficient is the structure sheaf.

\section{Review of Hyodo-Kato isomorphism.}

First, we briefly review the Hyodo-Kato isomorphism ([HK]). We also prepare some notations. Choose a uniformizer $\pi$ of $K$. Let $\left(V, M_{V}\right)$ be the scheme Spec $W[t]$ with $\log$-structure defined by the divisor " $t=0$ ". Let $F_{V}$ be the Frobenius lift defined by $t \mapsto t^{p}$ and $\sigma$. We have an $W$-exact closed immersion $i_{V, \pi}:(S, N) \hookrightarrow\left(V, M_{V}\right)$ defined by $t \mapsto \pi$. Let

$$
i_{E_{n}, \pi}:\left(S_{n}, N_{n}\right) \hookrightarrow\left(E_{n}, M_{E_{n}}\right)
$$

be the PD-envelope of $i_{V_{n}, \pi}:=\left(i_{V, \pi}\right)_{n}$. The lifting $F_{V_{n}}:=\left(F_{V}\right)_{n}$ induces a lifting of Frobenius $F_{E_{n}}:\left(E_{n}, M_{E_{n}}\right) \rightarrow\left(E_{n}, M_{E_{n}}\right)$ The $\operatorname{ring} R_{E_{n}}:=\Gamma\left(E_{n}, \mathcal{O}_{E_{n}}\right)$ is isomorphic 
to $W\left[t, t^{m e} / m !(m \geq 1)\right] \otimes_{W} W_{n}$, where $e:=\left[K: K_{0}\right]$. Put $R_{E}:=\lim _{n} R_{E_{n}}$. We also have an $W_{n}$-exact closed immersion $i_{V_{n}, 0}:\left(\operatorname{Spec} W_{n}, N_{n}^{0}\right) \hookrightarrow\left(V_{n}, M_{V_{n}}\right)$ defined by $t \mapsto 0$. It induces a $W_{n}$-exact closed immersion

$$
i_{E_{n}, 0}:\left(\operatorname{Spec} W_{n}, N_{n}^{0}\right) \hookrightarrow\left(E_{n}, M_{E_{n}}\right) .
$$

We have a commutative diagram of exact closed immersions:

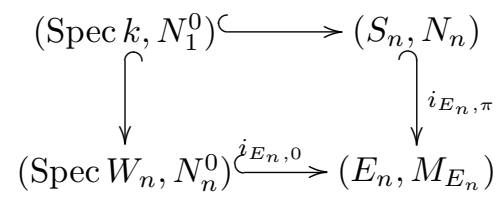

The morphism $i_{E_{n}, \pi}$ induces a homomorphism

$$
\operatorname{pr}_{\pi}: H_{\text {crys }}^{m}\left(\left(X_{n}, M_{n}\right) /\left(E_{n}, M_{E_{n}}\right)\right) \rightarrow H_{\text {crys }}^{m}\left(\left(X_{n}, M_{n}\right) /\left(S_{n}, N_{n}\right)\right) .
$$

The natural morphism $\left(Y, M_{Y}\right) \hookrightarrow\left(X_{n}, M_{n}\right)$ and $i_{E_{n}, 0}$ induce a homomorphism

$$
\operatorname{pr}_{0}: H_{\text {crys }}^{m}\left(\left(X_{n}, M_{n}\right) /\left(E_{n}, M_{E_{n}}\right)\right) \rightarrow H_{\text {crys }}^{m}\left(\left(Y, M_{Y}\right) /\left(W_{n}, N_{n}^{0}\right)\right) .
$$

Here we have

$$
H_{\text {crys }}^{m}\left(\left(X_{n}, M_{n}\right) /\left(E_{n}, M_{E_{n}}\right)\right) \cong H_{\text {crys }}^{m}\left(\left(X_{1}, M_{1}\right) /\left(E_{n}, M_{E_{n}}\right)\right)
$$

and $H_{\text {crys }}^{m}\left(\left(Y, M_{Y}\right) /\left(W_{n}, N_{n}^{0}\right)\right)$ are equipped with $\sigma$-semilinear endomorphism $\varphi$, which are induced by absolute Frobenius of $X_{1}$ and $Y$ and lifting of Frobenius $F_{E_{n}}$ and $\sigma$ respectively. Hyodo-Kato $([\mathrm{HK}])$ proved $\varphi$ is a bijection after $\mathbb{Q} \otimes \lim _{n}$ (for more general log-schemes) by using the theory of de Rham-Witt complex (In the case of crystalline cohomology, Berthelot-Ogus showed the analogous statement by the theory of "gauges". These bijections were essential for proving Berthelot-Ogus isomorphism and Hyodo-Kato isomorphism respectively). Put

$$
\begin{aligned}
H_{\text {crys }}^{m}\left(\left(Y, M_{Y}\right) /\left(W, N^{0}\right)\right) & :={\underset{\bigsqcup}{n}}_{\lim _{\text {crys }}} H^{m}\left(\left(Y, M_{Y}\right) /\left(W_{n}, N_{n}^{0}\right)\right), \\
H_{\text {crys }}^{m}((X, M) /(S, N)): & =\underbrace{\lim }_{n} H_{\text {crys }}^{m}\left(\left(X_{n}, M_{n}\right) /\left(S_{n}, N_{n}\right)\right), \\
H_{\text {crys }}^{m}\left((X, M) /\left(E, M_{E}\right)\right) & :=\underbrace{\lim }_{n} H_{\text {crys }}^{m}\left(\left(X_{n}, M_{n}\right) /\left(E_{n}, M_{E_{n}}\right)\right) .
\end{aligned}
$$

We have ([K1, Theorem (6.4)])

$$
H_{\text {crys }}^{m}\left(\left(X_{n}, M_{n}\right) /\left(S_{n}, N_{n}\right)\right) \cong \mathbb{H}^{m}\left(X_{n}, \Omega_{X_{n} / S_{n}}^{\bullet}\left(\log \left(M_{n} / N_{n}\right)\right)\right),
$$

where $\mathbb{H}$ means a hypercohomology of a complex. So, we have

$$
H_{\text {crys }}^{m}((X, M) /(S, N)) \cong \mathbb{H}^{m}\left(X, \Omega_{X / S}^{\bullet}(\log (M / N))\right)
$$

since $X$ is proper over $S$. Thus, we have

$$
\mathbb{Q} \otimes H_{\text {crys }}^{m}((X, M) /(S, N)) \cong \mathbb{H}^{m}\left(X_{K}, \Omega_{X_{K} / K}\right)=: H_{\mathrm{dR}}^{m}\left(X_{K} / K\right) .
$$

Lemma (5.2) of [HK] or Proposition 4.4.6 of [Tsu] tell us the existence of a $K_{0}$-linear section of $\mathbb{Q} \otimes \operatorname{pr}_{0}$ compatible with $\varphi$ :

$$
s: H_{\text {crys }}^{m}\left(\left(Y, M_{Y}\right) /\left(W, N^{0}\right)\right) \rightarrow \mathbb{Q} \otimes H_{\text {crys }}^{m}\left((X, M) /\left(E, M_{E}\right)\right),
$$


which induces an $R_{E}$-isomorphism:

$$
R_{E} \otimes_{W} H_{\text {crys }}^{m}\left(\left(Y, M_{Y}\right) /\left(W, N^{0}\right)\right) \stackrel{\sim}{\rightarrow} \mathbb{Q} \otimes H_{\text {crys }}^{m}\left((X, M) /\left(E, M_{E}\right)\right) .
$$

(Here, we need $\mathbb{Q} \otimes$ to get the section $s$, because we need the inverse of $\varphi^{r}$, where $r$ is an integer satisfying $\pi^{p^{r}} \in p O_{K}$. They used that the $r$-powered absolute Frobenius $F_{S_{1}}^{r}: S_{1} \rightarrow S_{1}$ factors through $S_{1} \stackrel{f}{\rightarrow}$ Spec $k \rightarrow S_{1}$, and $X_{1} \times{ }_{S_{1}, F_{X_{1}}^{r}} S_{1}$ is isomorphic to $Y \times_{\text {Spec } k, f} S_{1}$. See the proof of Lemma 2.5.) By using $s$ and $\mathbb{Q} \otimes \operatorname{pr}_{\pi}$, we have a homomorphism

$$
\mathbb{Q} \otimes \operatorname{pr}_{\pi} \circ s: H_{\text {crys }}^{m}\left(\left(Y, M_{Y}\right) /\left(W, N^{0}\right)\right) \rightarrow H_{\mathrm{dR}}^{m}\left(X_{K} / K\right) .
$$

the Hyodo-Kato theorem ([HK, Theorem (5.1)]) tells us that this induces a $K$ isomorphism

$$
\rho_{\pi}: K \otimes_{W} H_{\mathrm{crys}}^{m}\left(\left(Y, M_{Y}\right) /\left(W, N^{0}\right)\right) \stackrel{\sim}{\rightarrow} H_{\mathrm{dR}}^{m}\left(X_{K} / K\right)
$$

\section{2. log-crystalline and de Rham first Chern Classes.}

Secondly, I recall the definitions of crystalline first Chern class and de Rham Chern class.

Let $\left(\mathcal{O}_{X}^{\times}\right)^{\log }$ and $\left(\mathcal{O}_{Y}^{\times}\right)^{\log }$ denote $M^{\mathrm{gp}}$ and $M_{Y}^{\mathrm{gp}}$ respectively (they can be regarded as log-version of multiplicative group). $\mathrm{Put} \mathrm{Pic}^{\log }(X)$ and $\mathrm{Pic}^{\log }(Y)$ to be $H^{1}\left(X,\left(\mathcal{O}_{X}^{\times}\right)^{\log }\right)$ and $H^{1}\left(Y,\left(\mathcal{O}_{Y}^{\times}\right)^{\log }\right)$ respectively. The inclusions $\mathcal{O}_{X}^{\times} \hookrightarrow\left(\mathcal{O}_{X}^{\times}\right)^{\log }$ and $\mathcal{O}_{Y}^{\times} \hookrightarrow\left(\mathcal{O}_{Y}^{\times}\right)^{\log }$ induce $\operatorname{Pic}(X) \rightarrow \operatorname{Pic}^{\log }(X)$ and $\operatorname{Pic}(Y) \rightarrow \operatorname{Pic}^{\log }(Y)$ respectively. The inclusions $\mathcal{O}_{Y}^{\times} \hookrightarrow\left(\mathcal{O}_{Y}^{\times}\right)^{\log }$ and $\mathcal{O}_{X}^{\times} \hookrightarrow\left(\mathcal{O}_{X}^{\times}\right)^{\log }$ induce $\operatorname{Pic}(Y) \rightarrow \operatorname{Pic}^{\log }(Y)$ and $\operatorname{Pic}(X) \rightarrow \operatorname{Pic}^{\log }(X)$ respectively. By definition, we have $M^{\mathrm{gp}}=\mathcal{O}_{X_{K}}^{\times}$. So, $\operatorname{Pic}{ }^{\log }(X)$ is nothing but $\operatorname{Pic}\left(X_{K}\right)$. When we choose a uniformizer, we have an isomorphism $\left(\mathcal{O}_{Y}^{\times}\right)^{\log \cong j_{*}} \mathcal{O}_{Y \text { sm }}^{\times} \cdot \pi^{\mathbb{Z}}$, where $Y^{\mathrm{sm}}(\stackrel{j}{\hookrightarrow} Y)$ is the smooth locus of $Y$, and we regard $\pi(\neq 0)$ as living in $M_{Y}$, which goes to 0 in $\mathcal{O}_{Y}$. So, we have an isomorphism $\operatorname{Pic}^{\log }(Y) \cong \operatorname{Pic}\left(Y^{\mathrm{sm}}\right)$. This isomorphism is independent of the choice of $\pi$, since changing $\pi$ by $u \pi\left(u \in O_{K}^{\times}\right)$gives only a difference of principal divisor $(u)=0$. Let $\operatorname{NS}\left(X_{K}\right)$ be $\operatorname{Pic}\left(X_{K}\right) / \operatorname{Pic}^{0}\left(X_{K}\right)$, where $\operatorname{Pic}^{0}\left(X_{K}\right)$ is the isomorphism class of line bundles of algebraically equivalent to 0 . Let $\operatorname{Pic}^{\log , 0}(Y)$ be the pull back of $\operatorname{Pic}^{0}\left(Y^{\mathrm{sm}}\right)$

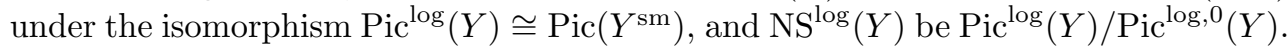
We call $\rho\left(X_{K}\right):=\operatorname{rankNS}\left(X_{K}\right)$, and $\rho(Y):=\operatorname{rankNS}^{\log }(Y)$, their Picard number. The homomorphism $\operatorname{Pic}\left(X_{K}\right)=\operatorname{Pic}^{\log }(X) \rightarrow \operatorname{Pic}^{\log }(Y)$ induces a homomorphism

$$
\mathrm{NS}\left(X_{K}\right) \rightarrow \mathrm{NS}^{\log }(Y) \text {. }
$$

Let $\left(\mathcal{O}_{\left(Y, M_{Y}\right) /\left(W_{n}, N_{n}^{0}\right)}^{\times}\right)^{\log }$ be a sheaf on the log-crystalline site

$$
\left(\left(Y, M_{Y}\right) /\left(W_{n}, N_{n}^{0}, p W_{n}, \gamma\right)\right)_{\text {crys }}^{\log }
$$

defined by $\left(\delta,\left(U,\left.M_{Y}\right|_{U}\right) \hookrightarrow\left(T, M_{T}\right)\right) \mapsto M_{T}^{\mathrm{gp}}$. Let

$$
i_{\left(Y, M_{Y}\right) /\left(W_{n}, N_{n}^{0}\right)}: Y_{\text {ét }} \rightarrow\left(\left(Y, M_{Y}\right) /\left(W_{n}, N_{n}^{0}\right)\right)_{\text {crys }}^{\log }
$$

be the canonical morphism (we use similar notations $i_{(-,-) /(-,-)}$for log-schemes). 
Lemma 2.1. We have exact sequences

$0 \rightarrow 1+J_{\left(Y, M_{Y}\right) /\left(W_{n}, N_{n}^{0}\right)} \rightarrow\left(\mathcal{O}_{\left(Y, M_{Y}\right) /\left(W_{n}, N_{n}^{0}\right)}^{\times}\right)^{\log } \rightarrow i_{\left(Y, M_{Y}\right) /\left(W_{n}, N_{n}^{0}\right), *}\left(\mathcal{O}_{Y}^{\times}\right)^{\log } \rightarrow 0$,

and

$$
0 \rightarrow 1+J_{\left(Y, M_{Y}\right) /\left(W_{n}, N_{n}^{0}\right)} \rightarrow \mathcal{O}_{\left(Y, M_{Y}\right) /\left(W_{n}, N_{n}^{0}\right)}^{\times} \rightarrow i_{\left(Y, M_{Y}\right) /\left(W_{n}, N_{n}^{0}\right), *} \mathcal{O}_{Y}^{\times} \rightarrow 0
$$

on $\left(\left(\left(Y, M_{Y}\right) /\left(W_{n}, N_{n}^{0}\right)\right)_{\text {crys }}^{\log }\right) \sim$.

Proof. The second one is classical. The first one comes from the exactness of the closed immersion $\left(U,\left.M_{Y}\right|_{U}\right) \hookrightarrow\left(T, M_{T}\right)$ for any

$$
\left(\delta,\left(U,\left.M_{Y}\right|_{U}\right) \hookrightarrow\left(T, M_{T}\right)\right) \in\left(\left(Y, M_{Y}\right) /\left(W_{n}, N_{n}^{0}\right)\right)_{\text {crys }}^{\log } .
$$

By using PD-structure on $J_{\left(Y, M_{Y}\right) /\left(W_{n}, N_{n}^{0}\right)}$, we have an isomorphism

$$
\log : 1+J_{\left(Y, M_{Y}\right) /\left(W_{n}, N_{n}^{0}\right)} \stackrel{\sim}{\rightarrow} J_{\left(Y, M_{Y}\right) /\left(W_{n}, N_{n}^{0}\right)} .
$$

So, by combining the boundary map of Lemma $2.1, \log$ and the inclusion

$$
i: J_{\left(Y, M_{Y}\right) /\left(W_{n}, N_{n}^{0}\right)} \hookrightarrow \mathcal{O}_{\left(Y, M_{Y}\right) /\left(W_{n}, N_{n}^{0}\right)},
$$

we get homomorphisms

$$
\begin{aligned}
\operatorname{Pic}^{\log }(Y) \stackrel{\partial}{\rightarrow} & H_{\text {crys }}^{2}\left(\left(Y, M_{Y}\right) /\left(W_{n}, N_{n}^{0}\right), 1+J_{\left(Y, M_{Y}\right) /\left(W_{n}, N_{n}^{0}\right)}\right) \\
& \stackrel{\log }{\cong} H_{\text {crys }}^{2}\left(\left(Y, M_{Y}\right) /\left(W_{n}, N_{n}^{0}\right), J_{\left(Y, M_{Y}\right) /\left(W_{n}, N_{n}^{0}\right)}\right) \\
& \stackrel{i}{\rightarrow} H_{\text {crys }}^{2}\left(\left(Y, M_{Y}\right) /\left(W_{n}, N_{n}^{0}\right)\right),
\end{aligned}
$$

and

$$
\begin{aligned}
\operatorname{Pic}(Y) \stackrel{\partial}{\rightarrow} & H_{\text {crys }}^{2}\left(\left(Y, M_{Y}\right) /\left(W_{n}, N_{n}^{0}\right), 1+J_{\left(Y, M_{Y}\right) /\left(W_{n}, N_{n}^{0}\right)}\right) \\
& \stackrel{\log }{\cong} H_{\text {crys }}^{2}\left(\left(Y, M_{Y}\right) /\left(W_{n}, N_{n}^{0}\right), J_{\left(Y, M_{Y}\right) /\left(W_{n}, N_{n}^{0}\right)}\right) \\
& \stackrel{i}{\rightarrow} H_{\text {crys }}^{2}\left(\left(Y, M_{Y}\right) /\left(W_{n}, N_{n}^{0}\right)\right) .
\end{aligned}
$$

We call them log-crystalline first Chern class homomorphism, and let $c_{\text {crys }}$ denote them. Here,

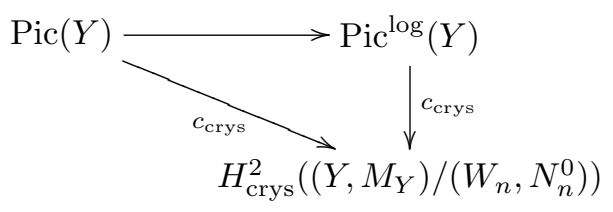

is commutative, because

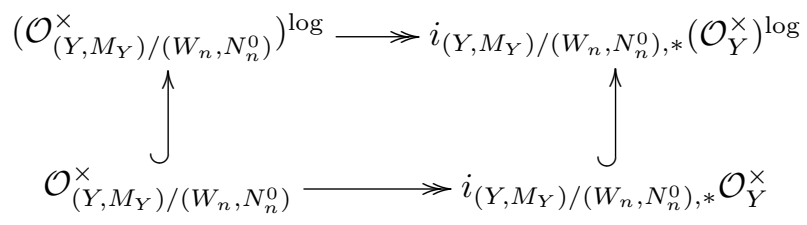

is commutative. 
Proposition 2.2. The image of the map

$$
c_{\text {crys }}: \operatorname{Pic}^{\log }(Y) \rightarrow H_{\text {crys }}^{2}\left(\left(Y, M_{Y}\right) /\left(W_{n}, N_{n}^{0}\right)\right)
$$

is in

$$
H_{\text {crys }}^{2}\left(\left(Y, M_{Y}\right) /\left(W_{n}, N_{n}^{0}\right)\right)^{\varphi=p, \mathcal{N}=0},
$$

where $\mathcal{N}$ is the monodromy operator on it ([HK, (3.5)], [Tsu, §4.4]).

Proof. First, we have

$$
\varphi\left(c_{\text {crys }}([L])\right)=c_{\text {crys }}(\varphi([L]))=c_{\text {crys }}\left(\left[L^{p}\right]\right)=c_{\text {crys }}(p[L])=p c_{\text {crys }}([L]) .
$$

Next, we will show that $\mathcal{N}\left(c_{\text {crys }}([L])\right)=0$. Let $\left(\operatorname{Spec} W[t], N_{W[t]}\right)$ be a log-scheme whose $\log$-structure is defined by " $t=0$ ", and $\left(\operatorname{Spec} W_{n}\langle t\rangle, N_{W_{n}\langle t\rangle}\right)$ be the PDenvelope of $\left(\operatorname{Spec} W_{n}, N_{n}^{0}\right) \hookrightarrow\left(\operatorname{Spec} W_{n}[t], N_{W_{n}[t]}\right)$. We take an embedding system $\left(Y^{\bullet},\left.M_{Y}\right|_{Y}\right) \hookrightarrow\left(Z \bullet, M_{Z}\right)$ for $\left(Y, M_{Y}\right)$ over $\left(\operatorname{Spec} W[t], N_{W[t]}\right)$. Let $\left(D_{n}^{\bullet}, M_{D_{\dot{n}}}\right)$ be the PD-envelope of $\left(Y^{\bullet},\left.M_{Y}\right|_{Y} \bullet \hookrightarrow\left(Z_{n}^{\bullet}, M_{Z_{n}^{\bullet}}\right)\right.$. Then, we have an exact sequence

$0 \rightarrow \mathcal{O}_{D_{n}^{\bullet}} \otimes_{\mathcal{O}_{Z_{n}}} \omega_{Z_{n}^{\bullet} / W_{n}\langle t\rangle}[-1] \stackrel{\wedge \operatorname{dlog}(t)}{\rightarrow} \mathcal{O}_{D_{n}^{\bullet}} \otimes_{\mathcal{O}_{Z_{n}}} \omega_{Z_{n} / W_{n}}^{\bullet} \rightarrow \mathcal{O}_{D_{n}^{\bullet}} \otimes_{\mathcal{O}_{Z_{n}}} \omega_{Z_{n}^{\bullet} / W_{n}\langle t\rangle}^{\bullet} \rightarrow 0$

on $\left(Y^{\bullet}\right)_{\text {ét }}^{\sim}$, where $\omega_{Z_{\dot{n}}^{\bullet} / W_{n}}^{\bullet}$ and $\omega_{Z_{\dot{n}} / W_{n}\langle t\rangle}^{\bullet}$ denote

$$
\Omega_{Z_{\dot{n}}^{\bullet} / W_{n}}^{\bullet}\left(\log \left(M_{Z_{n}^{\bullet}}\right)\right) \text { and } \Omega_{Z_{n}^{\bullet} / W_{n}\langle t\rangle}^{\bullet}\left(\log \left(M_{Z_{\dot{n}}} / N_{W_{n}\langle t\rangle}\right)\right)
$$

respectively. The boundary homomorphism associated to the above exact sequence after tensoring $W_{n}$ over $W_{n}\langle t\rangle$ with respect to $t^{[i]} \mapsto 0(i \geq 0)$ gives

$$
\begin{aligned}
H_{\text {crys }}^{m}\left(\left(Y, M_{Y}\right) /\left(W_{n}, \mathcal{O}_{W_{n}}^{\times}\right)\right) & \rightarrow H_{\text {crys }}^{m}\left(\left(Y, M_{Y}\right) /\left(W_{n}, N_{n}^{0}\right)\right) \\
& \stackrel{\partial}{\rightarrow} H_{\text {crys }}^{m}\left(\left(Y, M_{Y}\right) /\left(W_{n}, N_{n}^{0}\right)\right),
\end{aligned}
$$

where $\mathcal{O}_{W_{n}}^{\times}$is the trivial $\log$-structure on Spec $W_{n}$. The above boundary homomorphism $\partial$ is the monodromy operator ([HK, (3.6)], [K2, Lemma (4.2)])

$$
\mathcal{N}: H_{\text {crys }}^{m}\left(\left(Y, M_{Y}\right) /\left(W_{n}, N_{n}^{0}\right)\right) \rightarrow H_{\text {crys }}^{m}\left(\left(Y, M_{Y}\right) /\left(W_{n}, N_{n}^{0}\right)\right) .
$$

So, it suffices to show that there is a lift of

$$
c_{\text {crys }}: \operatorname{Pic}^{\log }(Y) \rightarrow H_{\text {crys }}^{2}\left(\left(Y, M_{Y}\right) /\left(W_{n}, N_{n}^{0}\right)\right)
$$

to $\operatorname{Pic}^{\log }(Y) \rightarrow H_{\text {crys }}^{2}\left(\left(Y, M_{Y}\right) /\left(W_{n}, \mathcal{O}_{W_{n}}^{\times}\right)\right)$. This can be done by a similar construction of $c_{\text {crys }}$, that is, the combination the boundary homomorphism of

$$
\begin{aligned}
0 \rightarrow 1+J_{\left(Y, M_{Y}\right) /\left(W_{n}, \mathcal{O}_{W_{n}}^{\times}\right)} & \rightarrow\left(\mathcal{O}_{\left(Y, M_{Y}\right) /\left(W_{n}, \mathcal{O}_{W_{n}}^{\times}\right)}^{\times}\right)^{\log } \\
& \rightarrow i_{\left(Y, M_{Y}\right) /\left(W_{n}, \mathcal{O}_{W_{n}}^{\times}\right), *}\left(\mathcal{O}_{Y}^{\times}\right)^{\log } \rightarrow 0,
\end{aligned}
$$

the isomorphism $1+J_{\left(Y, M_{Y}\right) /\left(W_{n}, \mathcal{O}_{W_{n}}^{\times}\right)} \stackrel{\log }{\cong} J_{\left(Y, M_{Y}\right) /\left(W_{n}, \mathcal{O}_{W_{n}}^{\times}\right)}$, and the inclusion

$$
J_{\left(Y, M_{Y}\right) /\left(W_{n}, \mathcal{O}_{W_{n}}^{\times}\right)} \hookrightarrow \mathcal{O}_{\left(Y, M_{Y}\right) /\left(W_{n}, \mathcal{O}_{W_{n}}^{\times}\right)} \cdot
$$


The commutativity of the following diagrams

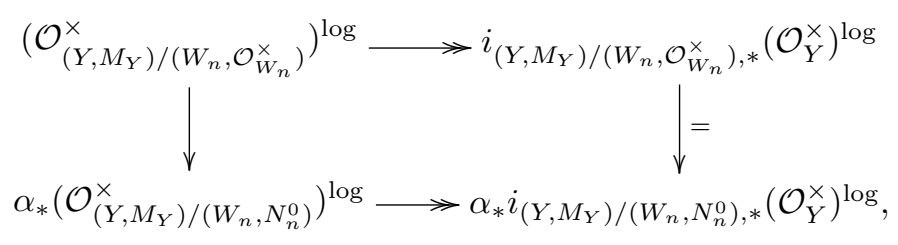

and

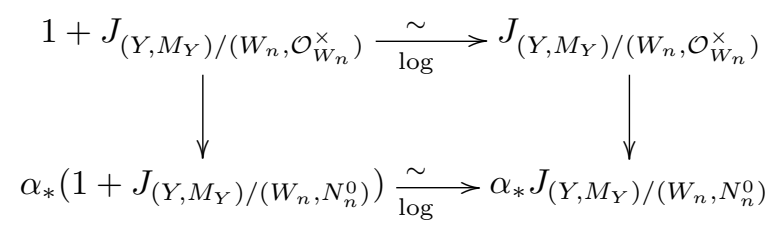

ensure that it's a lift of $c_{\text {crys }}$, where

$$
\alpha:\left(\left(Y, M_{Y}\right) /\left(W_{n}, N_{n}^{0}\right)\right)_{\text {crys }}^{\log } \rightarrow\left(\left(Y, M_{Y}\right) /\left(W_{n}, \mathcal{O}_{W_{n}}^{\times}\right)\right)_{\text {crys }}^{\log }
$$

is the morphism induced by the log-forgetting morphism

$$
\left(\operatorname{Spec} W_{n}, N_{n}^{0}\right) \rightarrow\left(\operatorname{Spec} W_{n}, \mathcal{O}_{W_{n}}^{\times}\right) .
$$

Corollary 2.3. For any $[L] \in \operatorname{Pic}^{\log }(Y)$,

$$
\rho_{\pi}\left(c_{\text {crys }}([L])\right) \in H_{\mathrm{dR}}^{2}\left(X_{K} / K\right)
$$

does not depend on the choice of $\pi$.

Proof. For any $u \in O_{K}^{\times}, \rho_{u \pi}$ is given by

$$
\rho_{u \pi}=\rho_{\pi} \circ \exp (\log (u) \mathcal{N})
$$

by $\left[H K\right.$, Theorem (5.1)] or [Tsu, Proposition 4.4.17]. Now, $\mathcal{N} c_{\text {crys }}([L])=0$ by the Proposition. We are done.

Next, let's recall the de Rham first Chern class. We have a homomorphism of complexes

$$
\mathcal{O}_{X_{K}}^{\times} \rightarrow F^{1} \Omega_{X_{K} / K}^{\bullet}[1] \rightarrow \Omega_{X_{K} / K}^{\bullet}[1]
$$

where the first map is given by $\mathcal{O}_{X_{K}}^{\times} \ni f \mapsto d f / f \in \Omega_{X_{K} / K}^{1}$, and $F^{1} \Omega_{X_{K} / K}^{\bullet}$ is the first Hodge filtration $\Omega_{X_{K} / K}^{\bullet 1}$. So, this homomorphism induces a homomorphism

$$
\operatorname{Pic}\left(X_{K}\right) \rightarrow H^{2}\left(X_{K}, F^{1} \Omega_{X_{K} / K}^{\bullet}\right) \rightarrow H_{\mathrm{dR}}^{2}\left(X_{K} / K\right) .
$$

The composite is the first de Rham chern class, and let $c_{\mathrm{d} R}$ denote it. 
Proposition 2.4. The following diagram is commutative:

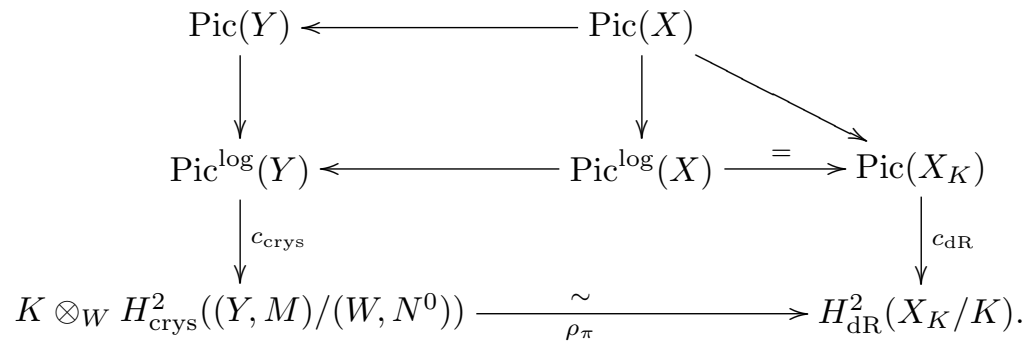

The non-trivial part is the bottom square.

We needs some preparations. Let $c_{\text {crys }}^{W_{n}}$ be the composition

$$
\operatorname{Pic}^{\log }(X) \rightarrow \operatorname{Pic}^{\log }(Y) \stackrel{c_{\text {crys }}}{\rightarrow} H_{\text {crys }}^{2}\left(\left(Y, M_{Y}\right) /\left(W_{n}, N_{n}^{0}\right)\right) .
$$

We can define

$$
c_{\text {crys }}^{E_{n}}: \operatorname{Pic}^{\log }(X) \rightarrow H_{\text {crys }}^{2}\left(\left(X_{n}, M_{n}\right) /\left(E_{n}, M_{E_{n}}\right)\right)
$$

and

$$
c_{\text {crys }}^{S_{n}}: \operatorname{Pic}^{\log }(X) \rightarrow H_{\text {crys }}^{2}\left(\left(X_{n}, M_{n}\right) /\left(S_{n}, N_{n}\right)\right)
$$

by the same way as $c_{\text {crys }}$. By the commutativity of the following diagrams

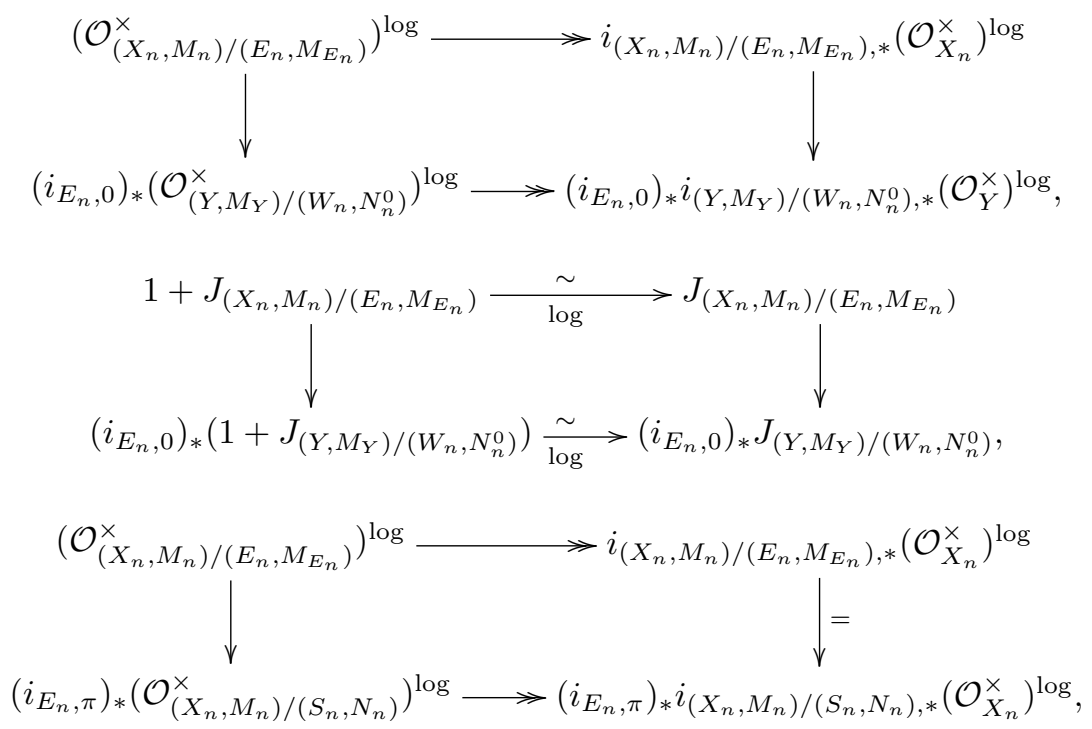

and

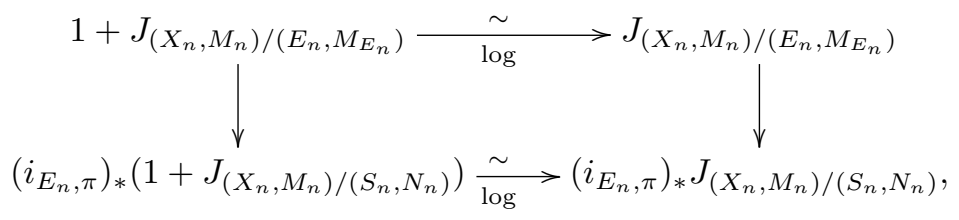


the following diagram is commutative:

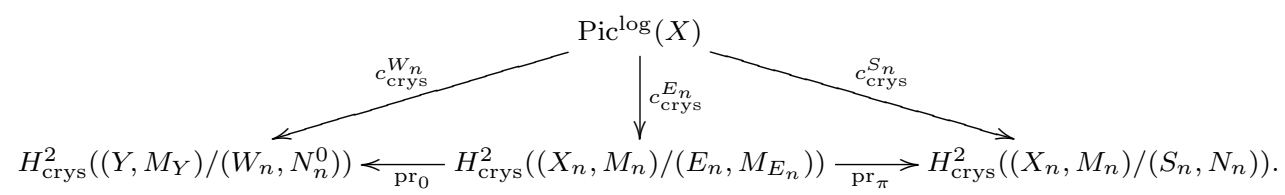

Here,

$$
i_{E_{n}, 0}:\left(\left(Y, M_{Y}\right) /\left(W_{n}, N_{n}^{0}\right)\right)_{\text {crys }}^{\log } \rightarrow\left(\left(X_{n}, M_{n}\right) /\left(E_{n}, M_{E_{n}}\right)\right)_{\text {crys }}^{\log }
$$

and

$$
i_{E_{n}, \pi}:\left(\left(X_{n}, M_{n}\right) /\left(S_{n}, N_{n}\right)\right)_{\text {crys }}^{\log } \rightarrow\left(\left(X_{n}, M_{n}\right) /\left(E_{n}, M_{E_{n}}\right)\right)_{\text {crys }}^{\log }
$$

are morphisms induced by $\left(Y, M_{Y}\right) \rightarrow\left(X_{n}, M_{n}\right)$ over $i_{E_{n}, 0}:\left(\operatorname{Spec} W_{n}, N_{n}^{0}\right) \hookrightarrow$ $\left(E_{n}, M_{E_{n}}\right)$ and $\left(X_{n}, M_{n}\right) \stackrel{\text { id }}{\longrightarrow}\left(X_{n}, M_{n}\right)$ over $i_{E_{n}, \pi}:\left(S_{n}, N_{n}\right) \hookrightarrow\left(E_{n}, M_{E_{n}}\right)$ respectively (we use the same symbols by the abuse of notations. the author hope that there would be no confusions).

Lemma 2.5. the following diagram is commutative:

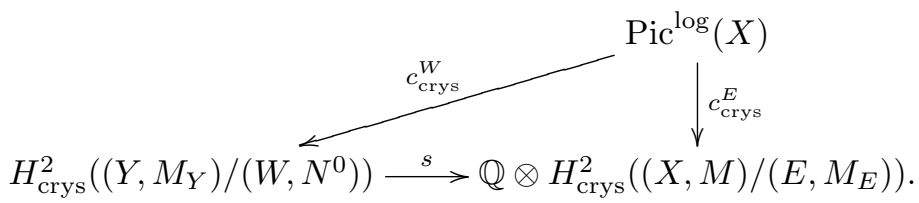

Proof. We recall the definition of the section $s$ ([HK, Lemma (5.2)], [Tsu, Proposition 4.4.6]). Let $r$ be an integer satisfying $\pi^{p^{r}} \in p O_{K}$. Then, the $r$-powered absolute Frobenius $F_{S_{1}}^{r}: S_{1} \rightarrow S_{1}$ factors through $S_{1} \stackrel{f}{\rightarrow}$ Spec $k \rightarrow S_{1}$. Thus, $X_{1} \times_{S_{1}, F_{X_{1}}^{r}} S_{1}$ is isomorphic to $Y \times \operatorname{Spec} k, f S_{1}$. Consider the following commutative diagram:

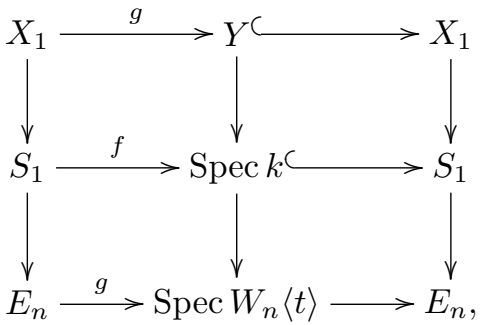

where $g$ is defined by $t \mapsto t^{p^{r}}$ and $\sigma^{r}$, and three composite horizontal arrows are $r$-powered absolute Frobenii. The left big squre with $g$ 's and the morphism

$$
\left(\operatorname{Spec} W_{n}\langle t\rangle, N_{W_{n}\langle t\rangle}\right) \rightarrow\left(\operatorname{Spec} W_{n}, N_{n}^{0}\right)
$$

defined by $t^{[n]} \mapsto 0$ induce

$$
g:\left(\left(X_{1}, M_{1}\right) /\left(E_{n}, M_{E_{n}}\right)\right)_{\text {crys }}^{\log } \rightarrow\left(\left(Y, M_{Y}\right) /\left(W_{n}\langle t\rangle, N_{W_{n}\langle t\rangle}\right)\right)_{\text {crys }}^{\log }
$$

and

$$
\beta:\left(\left(Y, M_{Y}\right) /\left(W_{n}\langle t\rangle, N_{W_{n}\langle t\rangle}\right)\right)_{\text {crys }}^{\log } \rightarrow\left(\left(Y, M_{Y}\right) /\left(W_{n}, N_{n}^{0}\right)\right)_{\text {crys }}^{\log }
$$

respectively (we use the same symbol $g$ by the abuse of notations). 
Then, we have isomorphisms ([HK, Lemma (5.2), Lemma (5.3)]):

$$
\begin{aligned}
& R_{E} \otimes_{W} H_{\text {crys }}^{m}\left(\left(Y, M_{Y}\right) /\left(W, N^{0}\right)\right)_{\mathbb{Q}} \stackrel{\sim, \varphi^{r}}{\longleftarrow} R_{E} \otimes_{\varphi^{r}, W} H_{\text {crys }}^{m}\left(\left(Y, M_{Y}\right) /\left(W, N^{0}\right)\right)_{\mathbb{Q}} \\
& \cong R_{E} \otimes_{g, W\langle t\rangle} H_{\text {crys }}^{m}\left(\left(Y, M_{Y}\right) /\left(W\langle t\rangle, N_{W\langle t\rangle}\right)\right)_{\mathbb{Q}} \\
& \cong R_{E} \otimes_{\varphi^{r}, R_{E}} H_{\text {crys }}^{m}\left(\left(X_{1}, M_{1}\right) /\left(E, M_{E}\right)\right)_{\mathbb{Q}} \\
& \stackrel{\sim, \varphi^{r}}{\longrightarrow} H_{\text {crys }}^{m}\left(\left(X_{1}, M_{1}\right) /\left(E, M_{E}\right)\right)_{\mathbb{Q}} \cong H_{\text {crys }}^{m}\left((X, M) /\left(E, M_{E}\right)\right)_{\mathbb{Q}},
\end{aligned}
$$

where the subscript $\mathbb{Q}$ means $\mathbb{Q} \otimes$. The composition of the above isomorphisms does not depend on $r$. The section $s$ is the composite of the above isomorphisms and $H_{\text {crys }}^{m}\left(\left(Y, M_{Y}\right) /\left(W, N^{0}\right)\right) \rightarrow R_{E} \otimes_{W} H_{\text {crys }}^{m}\left(\left(Y, M_{Y}\right) /\left(W, N^{0}\right)\right)_{\mathbb{Q}}$. By the same way as $c_{\text {crys }}$, we can define

$$
c_{\text {crys }}^{W_{n}\langle t\rangle}: \operatorname{Pic}^{\log }(X) \rightarrow H_{\text {crys }}^{2}\left(\left(Y, M_{Y}\right) /\left(W_{n}\langle t\rangle, N_{W\langle t\rangle}\right)\right) .
$$

So, it suffices to show that the following diagrams are commutative:

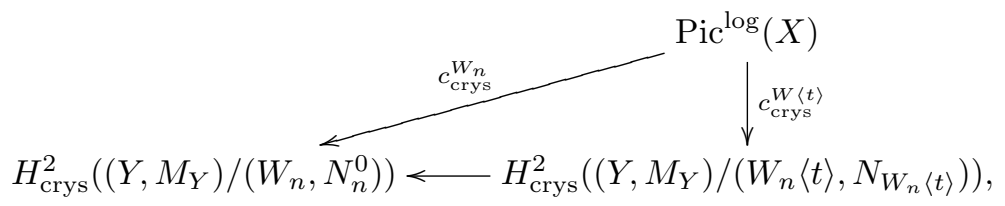

and

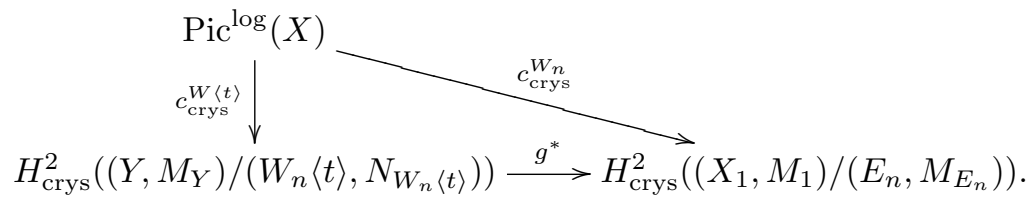

The commutativity of the diagrams follows from the commutativity of the following diagrams:

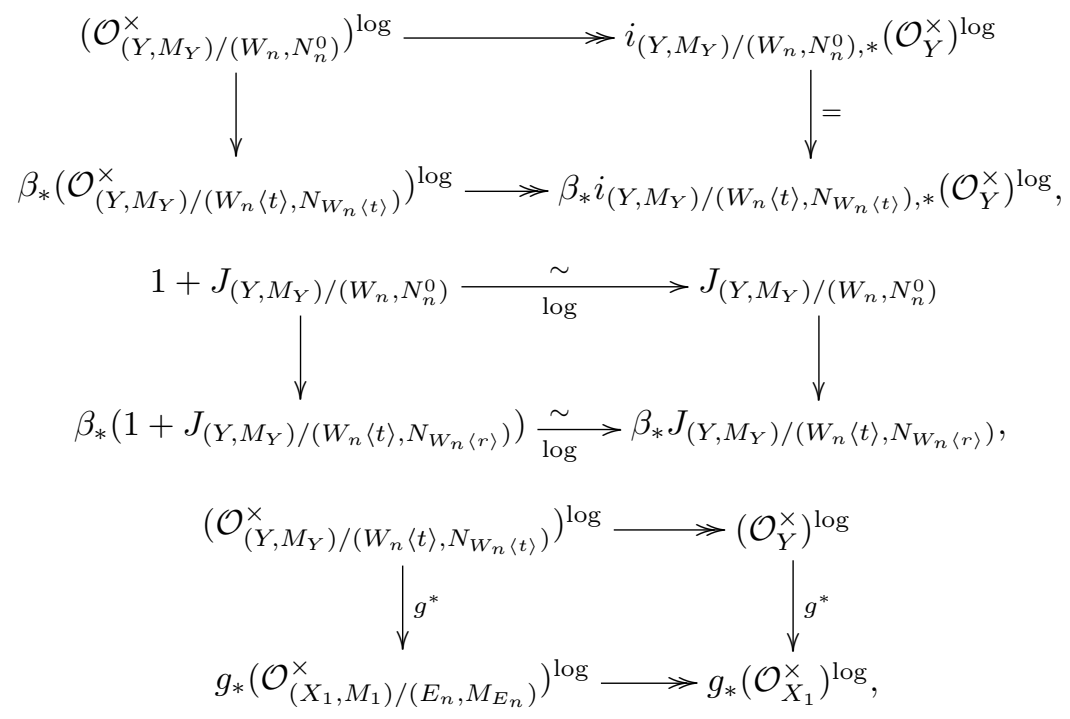


and

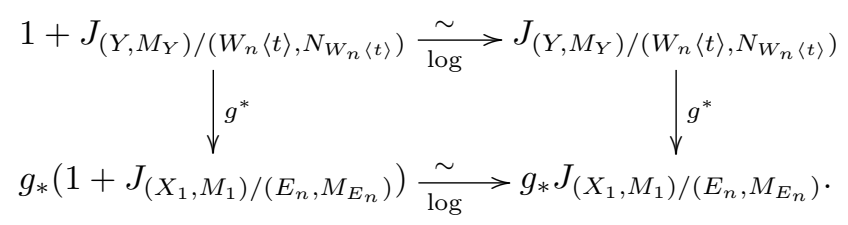

Proof. (Proof of Proposition 2.4) By Lemma 2.5, the following diagram is commutative:

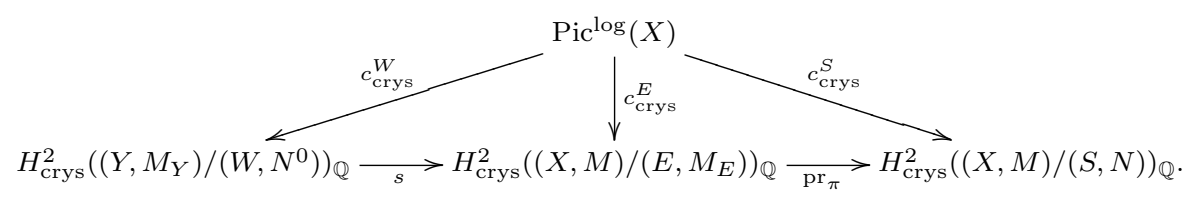

So, it suffices to show the following diagram is commutative (see [BO, Lemma (3.3), Proposition (3.4)] for the good reduction case):

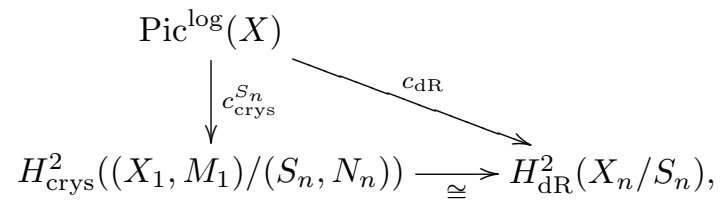

where $c_{\mathrm{dR}}$ is defined by

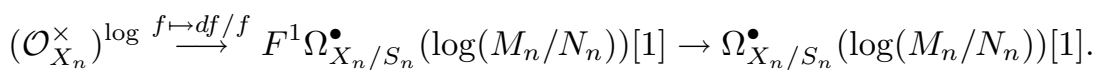

Put $\omega_{X_{n} / S_{n}}^{\bullet}:=\Omega_{X_{n} / S_{n}}^{\bullet}\left(\log \left(M_{n} / N_{n}\right)\right)$. Let $L\left(\omega_{X_{n} / S_{n}}^{\bullet}\right)$ be the complex on

$$
\left(\left(X_{1}, M_{1}\right) /\left(S_{n}, N_{n}\right)\right)_{\text {crys }}^{\log }
$$

deduced from $\omega_{X_{n} / S_{n}}^{\bullet}$ by linearization. We have a canonical homomorphism

$$
\mathcal{O}_{\left(X_{1}, M_{1}\right) /\left(S_{n}, N_{n}\right)} \rightarrow L\left(\mathcal{O}_{X_{n}}\right)
$$

and $L\left(\omega_{X_{n} / S_{n}}^{\bullet}\right)$ is a resolution of $\mathcal{O}_{\left(X_{1}, M_{1}\right) /\left(S_{n}, N_{n}\right)}$ by PD-Poincaré lemma. There is a surjective homomorphism $L\left(\mathcal{O}_{X_{n}}\right) \rightarrow \mathcal{O}_{X_{1}}$. Let $\mathcal{K}$ be the kernel. It is a PD-ideal in $L\left(\mathcal{O}_{X_{n}}\right)$ such that $\left(\mathcal{O}_{\left(X_{1}, M_{1}\right) /\left(S_{n}, N_{n}\right)}, J_{\left(X_{1}, M_{1}\right) /\left(S_{n}, N_{n}\right)}\right) \rightarrow\left(L\left(\mathcal{O}_{X_{n}}\right), \mathcal{K}\right)$ is a PDhomomorphism. Then $\mathcal{K}^{\times}$is the kernel of $L\left(\mathcal{O}_{X_{n}}\right)^{\times} \rightarrow \mathcal{O}_{X_{1}}^{\times}$, and it is isomorphic to the kernel of $L\left(\left(\mathcal{O}_{X_{n}}^{\times}\right)^{\log }\right) \rightarrow\left(\mathcal{O}_{X_{1}}^{\times}\right)^{\log }$, since $\left(U,\left.M_{1}\right|_{U}\right) \hookrightarrow\left(T, M_{T}\right)$ is exact for any $\left(\delta,\left(U,\left.M_{1}\right|_{U}\right) \hookrightarrow\left(T, M_{T}\right)\right) \in\left(\left(X_{1}, M_{1}\right) /\left(S_{n}, N_{n}\right)\right)_{\text {crys }}^{\log }$. Let $L\left(\omega_{X_{n} / S_{n}}^{\bullet}\right)^{\times}\left(\right.$resp. $\mathcal{K}^{\bullet}$, $\left.\mathcal{K}^{\bullet}, \times\right)$ denote the complex

$$
\begin{gathered}
L\left(\left(\mathcal{O}_{X_{n}}^{\times}\right)^{\log }\right) \stackrel{\operatorname{dlog}}{\rightarrow} L\left(\omega_{X_{n} / S_{n}}^{1}\right) \stackrel{L(d)}{\rightarrow} L\left(\omega_{X_{n} / S_{n}}^{2}\right) \rightarrow \cdots \\
\left(\text { resp. } \mathcal{K} \stackrel{L(d)}{\rightarrow} L\left(\omega_{X_{n} / S_{n}}^{1}\right) \stackrel{L(d)}{\rightarrow} L\left(\omega_{X_{n} / S_{n}}^{2}\right) \rightarrow \cdots,\right. \\
\left.1+\mathcal{K} \stackrel{\text { dlog }}{\rightarrow} L\left(\omega_{X_{n} / S_{n}}^{1}\right) \stackrel{L(d)}{\rightarrow} L\left(\omega_{X_{n} / S_{n}}^{2}\right) \rightarrow \cdots\right) .
\end{gathered}
$$


Then, we have the commutative diagrams:

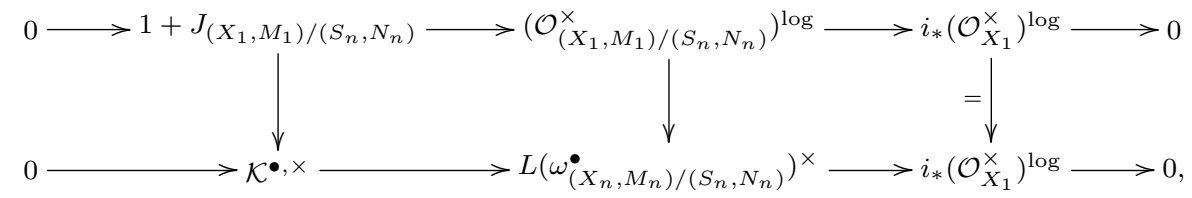

(where two $i_{*}$ 's mean $i_{\left(X_{1}, M_{1}\right) /\left(S_{n}, N_{n}\right), *}$ ) and

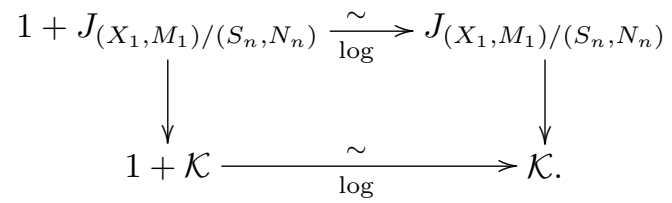

Thus, the following diagram is commutative:

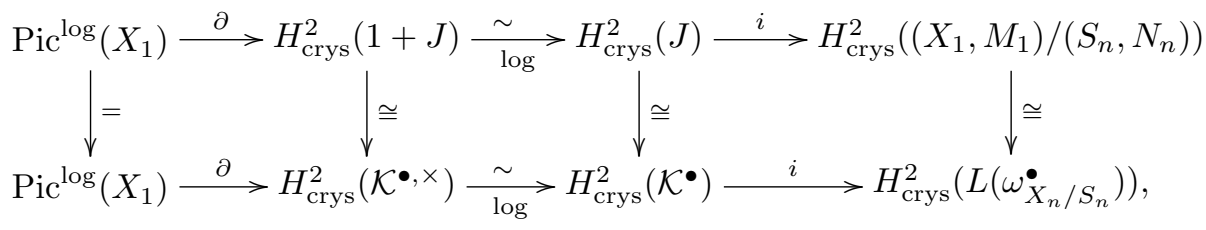

where $H_{\text {crys }}^{2}(-)$ 's mean $H_{\text {crys }}^{2}\left(\left(X_{1}, M_{1}\right) /\left(S_{n}, N_{n}\right),-\right)$, and $J$ means $J_{\left(X_{1}, M_{1}\right) /\left(S_{n}, N_{n}\right)}$. Here, the composition of the upper horizontal arrows is the definition of $c_{\text {crys }}^{S_{n}}$. Thus, $c_{\text {crys }}^{S_{n}}$ is equal to the composition of the lower horizontal arrows under the identification with $H_{\text {crys }}^{2}\left(\left(X_{1}, M_{1}\right) /\left(S_{n}, N_{n}\right)\right) \cong H_{\text {crys }}^{2}\left(L\left(\omega_{X_{n} / S_{n}}^{\bullet}\right)\right)$.

The kernel of $\mathcal{O}_{X_{n}}^{\times} \rightarrow \mathcal{O}_{X_{1}}^{\times}$is $1+p \mathcal{O}_{X_{n}}$, and this is also isomorphic to the kernel of $\left(\mathcal{O}_{X_{n}}^{\times}\right)^{\log } \rightarrow\left(\mathcal{O}_{X_{1}}^{\times}\right)^{\log }$. Let $J_{X_{n}, S_{n}}^{\bullet}\left(\operatorname{resp} . J_{X_{n}, S_{n}}^{\bullet, \times}\right)$ denote the complex

$$
\begin{gathered}
p \mathcal{O}_{X_{n}} \stackrel{d}{\rightarrow} \omega_{X_{n} / S_{n}}^{1} \stackrel{d}{\rightarrow} \omega_{X_{n} / S_{n}}^{2} \rightarrow \cdots \\
\text { (resp. } \left.1+p \mathcal{O}_{X_{n}} \stackrel{\text { dlog }}{\longrightarrow} \omega_{X_{n} / S_{n}}^{1} \stackrel{d}{\rightarrow} \omega_{X_{n} / S_{n}}^{2} \rightarrow \cdots\right) .
\end{gathered}
$$

Then, we have the following commutative diagram:

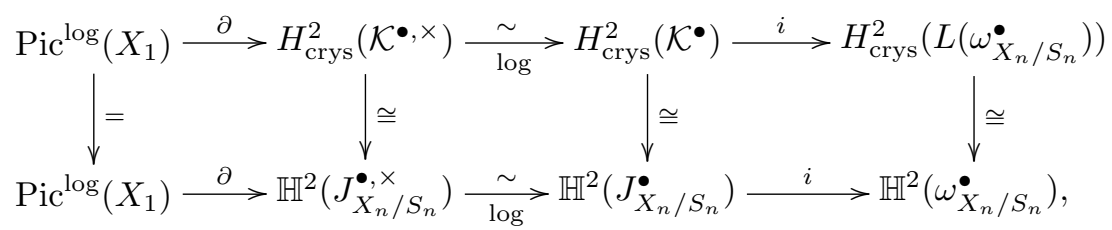

where $\mathbb{H}^{2}(-)$ 's mean $\mathbb{H}^{2}\left(X_{n},-\right)$ and $\partial$ in the bottom line is the boundary homomorphism induced by the exact sequence:

$$
0 \rightarrow J_{X_{n} / S_{n}}^{\bullet, \times} \rightarrow\left[\left(\mathcal{O}_{X_{n}}^{\times}\right)^{\log } \rightarrow \omega_{X_{n} / S_{n}}^{1} \rightarrow \omega_{X_{n} / S_{n}}^{1} \rightarrow \cdots\right] \rightarrow\left(\mathcal{O}_{X_{1}}^{\times}\right)^{\log } \rightarrow 0 .
$$

Thus, $c_{\text {crys }}^{S_{n}}$ is equal to the composition of the lower horizontal arrows under the identification with $H_{\text {crys }}^{2}\left(\left(X_{1}, M_{1}\right) /\left(S_{n}, N_{n}\right)\right) \cong H_{\text {crys }}^{2}\left(L\left(\omega_{X_{n} / S_{n}}^{\bullet}\right)\right) \cong \mathbb{H}^{2}\left(\omega_{X_{n} / S_{n}}^{\bullet}\right.$. 
We have the following commutative diagram whose horizontal lines are exact:

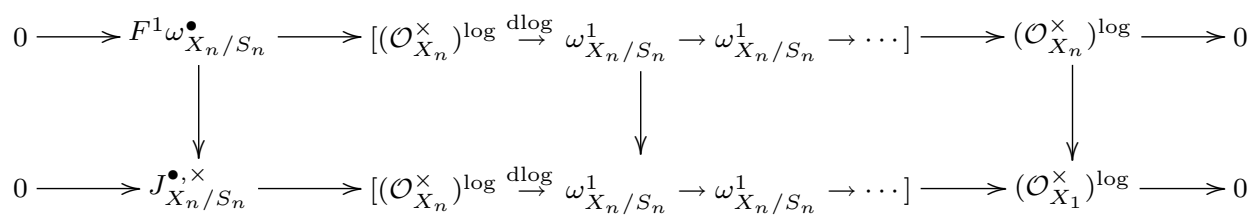

This gives the following commutative diagram:

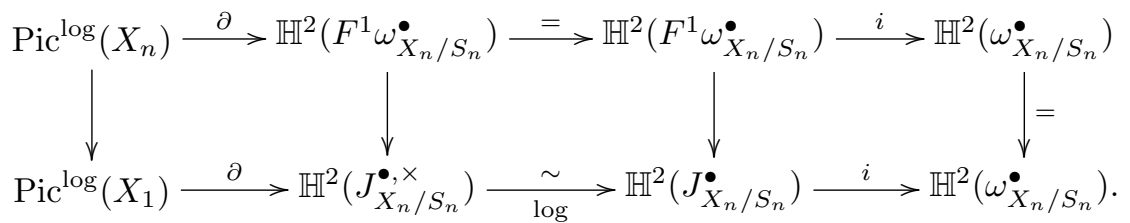

The composition of the upper horizontal arrows is the definition of $c_{\mathrm{dR}}$. We are done.

The following corollary is the semistable version of [BO, Corollary (3.7)].

Corollary 2.6. If $[L] \in \operatorname{Pic}^{\log }(Y)$ (resp. $[L] \in \operatorname{Pic}(Y)$ ) lifts to $\operatorname{Pic}^{\log }(X)$ (resp. $\operatorname{Pic}(X))$, then

is in $F^{1} H_{\mathrm{dR}}^{2}\left(X_{K} / K\right)$.

$$
\rho_{\pi}\left(c_{\text {crys }}([L])\right) \in H_{\mathrm{dR}}^{2}\left(X_{K} / K\right)
$$

\section{3. $p$-adic Lefschetz $(1,1)$ theorem in semistable case.}

The following theorem is the semistable version of [BO, Theorem (3.8)].

Theorem 3.1. The element $[L] \in \operatorname{Pic}^{\log }(Y)_{\mathbb{Q}}$ (resp. $\left.[L] \in \operatorname{Pic}(Y)_{\mathbb{Q}}\right)$ lifts to

$$
\operatorname{Pic}^{\log }(X)_{\mathbb{Q}},\left(\text { resp. } \operatorname{Pic}(X)_{\mathbb{Q}}\right) \text {, }
$$

if and only if

is in $F^{1} H_{\mathrm{dR}}^{2}\left(X_{K} / K\right)$.

$$
\rho_{\pi}\left(c_{\text {crys }}([L])\right) \in H_{\mathrm{dR}}^{2}\left(X_{K} / K\right)
$$

Lemma 3.2. The element $[L] \in \operatorname{Pic}^{\log }(Y)_{\mathbb{Q}}\left(\right.$ resp. $\left.[L] \in \operatorname{Pic}(Y)_{\mathbb{Q}}\right)$ lifts to $\operatorname{Pic}^{\log }\left(X_{1}\right)_{\mathbb{Q}}$ $\left(\right.$ resp. $\left.\operatorname{Pic}\left(X_{1}\right)_{\mathbb{Q}}\right)$.

Proof. Let $r$ be an integer satisfying $\pi^{p^{r}} \in p O_{K}$. Then, the $r$-powered absolute Frobenius $F_{S_{1}}^{r}: S_{1} \rightarrow S_{1}$ (resp. $F_{X_{1}} X_{1} \rightarrow X_{1}$ ) factors through $S_{1} \stackrel{f}{\rightarrow}$ Spec $k \rightarrow S_{1}$ (resp. $X_{1} \stackrel{g}{\rightarrow} Y \rightarrow X_{1}$ ). Thus, $X_{1} \times_{S_{1}, F_{X_{1}}^{r}} S_{1}$ is isomorphic to $Y \times_{\operatorname{Spec} k, f} S_{1}$. So, $g^{*}[L] \in \operatorname{Pic}^{\log }\left(X_{1}\right)$ (resp. $\left.g^{*}[L] \in \operatorname{Pic}^{\log }\left(X_{1}\right)\right)$ is a lift of $\left[L^{p^{r}}\right]$. Inverting $p^{r}$, we get a lift.

Proof. (Proof of Theorem 3.1) The direction of "only if" is Corollary 2.6. We show the other direction. Assume $\rho_{\pi}\left(c_{\text {crys }}([L])\right)$ is in $F^{1} H_{\mathrm{dR}}^{2}\left(X_{K} / K\right)$. By Lemma 3.2, we can take a lift $\left[L^{\prime}\right]$ of $[L]$ in $\operatorname{Pic}^{\log }\left(X_{1}\right)_{\mathbb{Q}}\left(\operatorname{resp}\right.$. $\left.\operatorname{Pic}\left(X_{1}\right)_{\mathbb{Q}}\right)$. Then by the commutative diagram (2) in the proof of Proposition 2.4, the image of $c_{\text {crys }}^{S}\left(\left[L^{\prime}\right]\right)$ in $H_{\mathrm{dR}}^{2}\left(X_{K} / K\right)$ is in $F^{1} H_{\mathrm{dR}}^{2}\left(X_{K} / K\right)$. By multiplying suitable integer, we can assume that the image of 
$c_{\text {crys }}^{S}\left(\left[L^{\prime}\right]\right)$ in $H_{\mathrm{dR}}^{2}\left(X_{K} / K\right) \cong H_{\mathrm{dR}}^{2}(X / S)_{\mathbb{Q}}$ comes from $F^{1} H_{\mathrm{dR}}^{2}(X / S)$. By the following commutative diagram

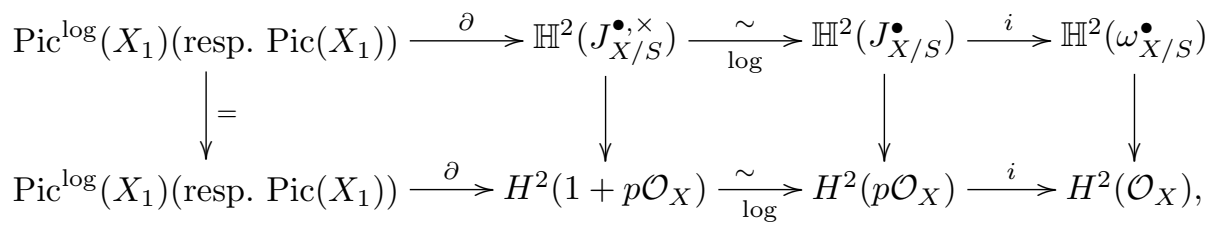

the image of $c_{\text {crys }}^{S}\left(\left[L^{\prime}\right]\right)=i \circ \log \circ \partial\left(\left[L^{\prime}\right]\right)$ in $H^{2}\left(X, \mathcal{O}_{X}\right)$ is zero, where $H^{2}(-)^{\prime}$ 's in the lower horizontal line mean $H^{2}(X,-)$. The second log in the above diagram is the comoposite

$$
H^{2}\left(X, 1+p \mathcal{O}_{X}\right) \cong H^{2}\left(\widehat{X}, 1+p \mathcal{O}_{\widehat{X}}\right) \stackrel{\log , \sim}{\longrightarrow} H^{2}\left(\widehat{X}, p \mathcal{O}_{\widehat{X}}\right) \cong H^{2}\left(X, p \mathcal{O}_{X}\right),
$$

where $\widehat{X}$ is the $p$-adic formal completion of $X$, and we used formal GAGA twice here. The composition

$$
p \mathcal{O}_{X} \hookrightarrow \mathcal{O}_{X} \stackrel{p, \sim}{\longrightarrow} p \mathcal{O}_{X}
$$

is the multiplication by $p$. Thus, the image of $p \cdot \log \circ \partial\left(\left[L^{\prime}\right]\right)$ in $H^{2}\left(X, p \mathcal{O}_{X}\right)$ is zero. The homomorphism log is an isomorphism, so the image of $p \partial\left(\left[L^{\prime}\right]\right)$ in $H^{2}\left(X, 1+p \mathcal{O}_{X}\right)$ is zero. On the other hand, the exact sequence

$$
\begin{gathered}
0 \rightarrow 1+p \mathcal{O}_{X} \rightarrow\left(\mathcal{O}_{X}^{\times}\right)^{\log } \rightarrow\left(\mathcal{O}_{X_{1}}^{\times}\right)^{\log } \rightarrow 0 \\
\left(\text { resp. } 0 \rightarrow 1+p \mathcal{O}_{X} \rightarrow \mathcal{O}_{X}^{\times} \rightarrow \mathcal{O}_{X_{1}}^{\times} \rightarrow 0\right)
\end{gathered}
$$

induces an exact sequence

$$
\begin{gathered}
\operatorname{Pic}^{\log }(X) \rightarrow \operatorname{Pic}^{\log }\left(X_{1}\right) \rightarrow H^{2}\left(X, 1+p \mathcal{O}_{X}\right) \\
\left(\text { resp. } \operatorname{Pic}(X) \rightarrow \operatorname{Pic}\left(X_{1}\right) \rightarrow H^{2}\left(X, 1+p \mathcal{O}_{X}\right)\right) .
\end{gathered}
$$

Here, $p\left[L^{\prime}\right]$ goes to zero in $H^{2}\left(X, 1+p \mathcal{O}_{X}\right)$. Therefore, $p\left[L^{\prime}\right]$ comes from $\operatorname{Pic}^{\log }(X)$ $($ resp. Pic $(X))$. So, $\left[L^{\prime}\right]$ comes from $\operatorname{Pic}^{\log }(X)_{\mathbb{Q}}\left(\operatorname{resp} . \operatorname{Pic}(X)_{\mathbb{Q}}\right)$.

\section{An application to Picard number jumping locus.}

We consider a generalization of the Maulik-Poonen result ([MP]).

First, we set up a situation. Let $C$ be the completion of an algebraic closure of $K$, and $O_{C}$ be its valuation ring. Let $B$ be an irreducible separated $O_{K}$-scheme of finite type, and let $f: \mathcal{X} \rightarrow B$ be a proper semistable morphism such that $f_{K}: \mathcal{X}_{K} \rightarrow B_{K}$ is smooth, where ()$_{K}$ 's mean $K \otimes_{O_{K}}$. Let $M_{\mathcal{X}}$ and $M_{B}$ be log-structures on $\mathcal{X}$ and $B$ defined by $\mathcal{X} \otimes_{O_{K}} k$ and $B \otimes_{O_{K}} k$ respectively. Let $s, t \in B$ be such that $s$ is a specialization of $t$ (i.e., $s$ is in the closure of $\{t\}$ ), $\operatorname{char} \kappa(t)=0$ and $\operatorname{char} \kappa(s)=p$. Let $\left(\mathcal{X}_{\bar{t}}, M_{\mathcal{X}_{\bar{t}}}\right)$ and $\left(\mathcal{X}_{\bar{s}}, M_{\mathcal{X}_{\bar{s}}}\right)$ be the fiber of $\left(\mathcal{X}, M_{\mathcal{X}}\right)$ at $\bar{t}$ and $\bar{s}$ respectively. By the same way as homomorphism (1), we have a homomorphism

$$
\operatorname{sp}_{\bar{t}, \bar{s}}: \operatorname{NS}\left(\mathcal{X}_{\bar{t}}\right) \rightarrow \mathrm{NS}^{\log }\left(\mathcal{X}_{\bar{s}}\right)
$$

Lemma 4.1. (1) The homomorphism

$$
\mathbb{Z}[1 / p] \otimes \operatorname{sp}_{\bar{t}, \bar{s}}: \operatorname{NS}\left(\mathcal{X}_{\bar{t}}\right)[1 / p] \rightarrow \mathrm{NS}^{\log }\left(\mathcal{X}_{\bar{s}}\right)[1 / p] .
$$

is injective, and its cokernel is torsion-free.

(2) We have $\rho\left(\mathcal{X}_{\bar{t}}\right) \geq \rho\left(\mathcal{X}_{\bar{s}}\right)$. 
(3) If $\operatorname{sp}_{\bar{t}, \bar{s}}$ maps a class $[L]$ to an ample class, then $L$ is ample.

Proof. These can be shown by a similar way as [MP, Proposition 3.6 (b), (c), (d)]. We will give a rough sketch here. For the details, see [MP, Proposition 3.6]. By using the following diagram (here, we replaced $H_{\text {ét }}^{2}\left(\mathcal{X}_{\bar{t}}, \mathbb{Z}_{\ell}(1)\right) \cong H_{\text {êt }}^{2}\left(\mathcal{X}_{\bar{s}}, \mathbb{Z}_{\ell}(1)\right)$ by $H_{\text {êt }}^{2}\left(\mathcal{X}_{\bar{t}}, \mathbb{Z}_{\ell}(1)\right) \cong H_{\text {log-ét }}^{2}\left(\left(\mathcal{X}_{\bar{s}}, M_{\mathcal{X}_{\bar{s}}}\right), \mathbb{Z}_{\ell}(1)\right)$ (see, $\left.\left.[\mathrm{N}]\right)\right)$ for any $\ell \neq p$ :

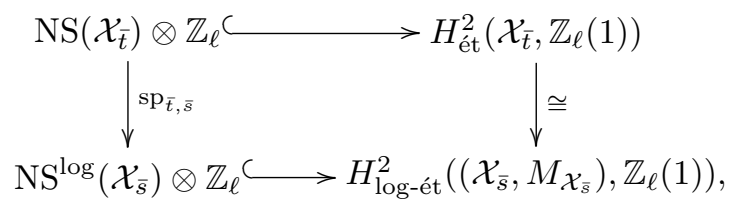

we have

$$
\operatorname{coker}\left(\operatorname{sp}_{\bar{t}, \bar{s}} \otimes \mathbb{Z}_{\ell}\right) \subset \operatorname{coker}\left\{\operatorname{NS}\left(\mathcal{X}_{\bar{t}}\right) \otimes \mathbb{Z}_{\ell} \rightarrow H_{\text {ét }}^{2}\left(\mathcal{X}_{\bar{t}}, \mathbb{Z}_{\ell}(1)\right)\right\} \cong T_{\ell} \operatorname{Br} \mathcal{X},
$$

where the last term is automatically torsion-free. This induces part (1) and (2). The part (3) comes from [MP, Proposition 3.3], which is essentially [EGA III.I, 4.7.1].

Let $M_{B_{k}}$ be the pull-back of $M_{B}$ on $B_{k}$. For a $p$-adic formal $O_{K}$-log-scheme $\left(T, M_{T}\right)$, let $T_{1}$ be the closed subscheme defined by $p \mathcal{O}_{T}$, and let $T_{0}$ be the associated reduced subscheme $\left(T_{1}\right)_{\text {red }}$. Let $M_{T_{1}}$ and $M_{T_{0}}$ be the pull-back of $M_{T}$ to $T_{1}$ and $T_{0}$ respectively. The notion of enlargement of [O, Definition 2.1] is generalized to the semistable case by Shiho [S, Definition 2.1.1]. We use his definition (note that we use fine $\log$ (formal) schemes, not fine saturated log (formal) schemes in his definition). By the same way as [O, Theorem 3.1 and 3.7], we can show that for any $q \in \mathbb{Z}_{\geq 0}$, there exists a log-convergent isocrystal $E:=R^{q} f_{\text {crys* }} \mathcal{O}_{\left(\mathcal{X}, M_{\mathcal{X}}\right) /\left(W, N^{0}\right)} \otimes_{W} K$ on $B_{k}$ with isomorphism of $K$-vector spaces

$$
E_{[s]} \cong H_{\text {crys }}^{q}\left(\left(\mathcal{X}_{s}, M_{\mathcal{X}_{s}}\right) /\left(W, N^{0}\right)\right) \otimes_{W} K
$$

for each $s \in B(k)$, where $[s]$ is a enlargement given by $\left(\left(\operatorname{Spf} O_{K}, N\right),\left(\operatorname{Spec} k, N_{1}^{0}\right) \stackrel{s}{\rightarrow}\right.$ $\left.\left(B_{k}, M_{B_{k}}\right)\right)$.

Proposition 4.2. Let $((T, M), z)$ be an enlargement of $\left(B_{k}, M_{B_{k}}\right)$. Let

$$
f_{0}:\left(\mathcal{X}_{0}, M_{\mathcal{X}_{0}}\right) \rightarrow\left(T_{0}, M_{T_{0}}\right)
$$

be obtained from $f:\left(\mathcal{X}, M_{\mathcal{X}}\right) \rightarrow\left(B, M_{B}\right)$ by base change along $z: T_{0} \rightarrow B_{k} \hookrightarrow B$. Let $g: \mathcal{Y} \rightarrow T$ be a proper semistable lifting of $f_{0}$. Let $M_{\mathcal{Y}}$ be the log-structure defined by $\mathcal{Y} \otimes_{O_{K}} k$. Then for each $q \in \mathbb{Z}_{\geq 0}$, there is a canonical isomorphism

$$
\left(R^{q} f_{\text {crys } *} \mathcal{O}_{\left(\mathcal{X}_{0}, M_{\mathcal{X}_{0}}\right) /\left(W, N^{0}\right)} \otimes_{W} K\right)_{\left(T, M_{T}\right)} \cong K \otimes R^{q} g_{*} \omega_{\mathcal{Y} / T},
$$

where $\omega_{\mathcal{Y} / T}^{\bullet}$ denotes $\Omega_{\mathcal{Y} / T}^{\bullet}\left(\log \left(M_{\mathcal{Y}} / M_{T}\right)\right)$.

Proof. We may assume that we have a Frobenius lift $F_{T}$ and $F_{\mathcal{Y}}$ on $T$ and $\mathcal{Y}$ respectively, since the problem is local. Let $r$ be an integer satisfying $\pi^{p^{r}} \in p O_{K}$. Then, the $r$-powered absolute Frobenius $F_{S_{1}}^{r}: S_{1} \rightarrow S_{1}$ factors through $S_{1} \stackrel{h}{\rightarrow}$ Spec $k \rightarrow$ 
$S_{1}$. Thus, $\mathcal{Y}_{0} \times_{S_{1}, F_{Y_{1}}^{r}} S_{1}$ and $T_{1} \times_{S_{1}, F_{T_{1}}^{r}} S_{1}$ are isomorphic to $\mathcal{X}_{0} \times_{\text {Spec } k, h} S_{1}$ and $T_{0} \times{ }_{\text {Spec } k, h} S_{1}$ respectively. Consider the following commutative diagram:

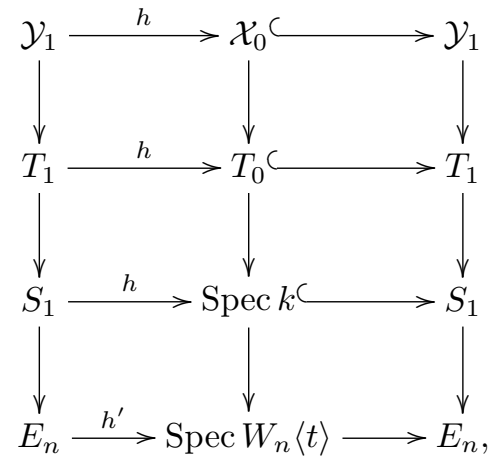

where $h^{\prime}$ is defined by $t \mapsto t^{p^{r}}$ and $\sigma^{r}$, and three composite horizontal arrows are $r$-powered absolute Frobenii. The composition of the above isomorphisms does not depend on $r$. Then, we have isomorphisms

$$
\begin{aligned}
& R_{E} \otimes_{W} R^{q} f_{\text {crys* }} \mathcal{O}_{\left(\mathcal{X}_{0}, M_{\mathcal{X}_{0}}\right) /\left(W, N^{0}\right)} \otimes \mathbb{Q} \stackrel{\sim, \varphi^{r}}{\longleftarrow} R_{E} \otimes_{\varphi^{r}, W} R^{q} f_{\text {crys* }} \mathcal{O}_{\left(\mathcal{X}_{0}, M_{\mathcal{X}_{0}}\right) /\left(W, N^{0}\right)} \otimes \mathbb{Q} \\
& \cong R_{E} \otimes_{h, W\langle t\rangle} R^{q} f_{\text {crys } *} \mathcal{O}_{\left(\mathcal{X}_{0}, M_{\mathcal{X}_{0}}\right) /\left(W\langle t\rangle, N_{W\langle t\rangle}\right)} \otimes \mathbb{Q} \\
& \cong R_{E} \otimes_{\varphi^{r}, R_{E}} R^{q} f_{\text {crys } *} \mathcal{O}_{\left(\mathcal{Y}_{1}, M_{\mathcal{Y}_{1}}\right) /\left(E, M_{E}\right)} \otimes \mathbb{Q} \\
& \stackrel{\sim, \varphi^{r}}{\longrightarrow} R^{q} f_{\text {crys } *} \mathcal{O}_{\left.\left(\mathcal{Y}_{1}, M_{\mathcal{Y}_{1}}\right) /\left(E, M_{E}\right)\right)} \otimes \mathbb{Q} \cong R^{q} f_{\text {crys } *} \mathcal{O}_{\left(\mathcal{Y}_{n}, M_{\mathcal{Y}_{n}}\right) /\left(E, M_{E}\right)} \otimes \mathbb{Q} .
\end{aligned}
$$

This induces the following isomorphism:

$$
\begin{aligned}
& \left(R^{q} f_{\text {crys } *} \mathcal{O}_{\left(\mathcal{X}_{0}, M_{\mathcal{X}_{0}}\right) /\left(W, N^{0}\right)} \otimes_{W} K\right)_{\left(T, M_{T}\right)} \cong\left(R^{q} f_{\text {crys } *} \mathcal{O}_{\left(\mathcal{Y}_{n}, M_{\mathcal{Y}_{n}}\right) /\left(E, M_{E}\right)} \otimes_{W} K\right)_{\left(T, M_{T}\right)} \\
& \cong\left(R^{q} f_{\text {crys } *} \mathcal{O}_{\left(\mathcal{Y}_{n}, M_{\mathcal{Y}_{n}}\right) /(S, N)} \otimes_{W} K\right)_{\left(T, M_{T}\right)} \\
& \cong \varliminf_{n}\left(R^{q} f_{\text {crys } *} \mathcal{O}_{\left(\mathcal{Y}_{n}, M_{\mathcal{Y}_{n}}\right) /(S, N)} \otimes_{W} K\right)_{\left(T, M_{T}\right)} \cong K \otimes R^{q} g_{*} \omega_{\mathcal{Y} / T} .
\end{aligned}
$$

The following theorem is a semistable version of the Maulik-Poonen result.

Theorem 4.3. The set

$$
B\left(O_{C}\right)_{\text {jumping }}:=\left\{b \in B\left(O_{C}\right) \mid \rho\left(\mathcal{X}_{b} \otimes_{O_{C}} \bar{k}\right)>\rho\left(\mathcal{X}_{\bar{\eta}}\right)\right\}
$$

is nowhere dense in $B\left(O_{C}\right)$ for the analytic topology.

Proof. It can be shown by the same way as [MP, Theorem 1.7]. Here, we have to replace [MP, Theorem 4.24] and [MP, Theorem 4.21] by Theorem 3.1 and Proposition 4.2 respectively. We will give a rough idea here.

Let $E$ be the above log-convergent isocrystal $E$ for $q=2$. We have the canonical isomorphism

$$
E_{[s]} \cong H_{\text {crys }}^{2}\left(\left(\mathcal{X}_{s}, M_{\mathcal{X}_{s}}\right) /\left(W, N^{0}\right)\right) \otimes_{W} K
$$

for each $s \in B(k)$, Take $\left[L_{k}\right] \in \operatorname{Pic}^{\log }\left(\mathcal{X}_{s}\right)$. Then

$$
c_{\text {crys }}\left(\left[L_{k}\right]\right) \in H_{\text {crys }}^{2}\left(\left(\mathcal{X}_{s}, M_{\mathcal{X}_{s}}\right) /\left(W, N^{0}\right)\right) \otimes_{W} K
$$


gives rise to a constant section $\gamma_{\text {crys }}\left(\left[L_{k}\right]\right)_{T}$ of $H_{\text {crys }}^{2}\left(\left(\mathcal{X}_{s}, M_{\mathcal{X}_{s}}\right) /\left(W, N^{0}\right)\right) \otimes_{W} \mathcal{O}_{T} \cong E_{T}$ for a morphism of enlargement $T \rightarrow[s]$. By using Proposition 4.2, this gives a section $\gamma_{\mathrm{dR}}\left(\left[L_{k}\right]\right)_{T}$ of $K \otimes R^{2} f_{*} \omega_{\left(\mathcal{X}_{T}, M_{\mathcal{X}_{T}}\right) /\left(T, M_{T}\right)}$, which can be mapped to a section $\gamma_{02}\left(\left[L_{k}\right]\right)_{T}$ of the quotient sheaf $K \otimes R^{2} f_{*} \omega_{\left(\mathcal{X}_{T}, M_{\mathcal{X}_{T}}\right) /\left(T, M_{T}\right)} / \mathrm{Fil}^{1}$. We can "evaluate" $\gamma_{\text {crys }}\left(\left[L_{k}\right]\right)_{T}, \gamma_{\mathrm{dR}}\left(\left[L_{k}\right]\right)_{T}$, and $\gamma_{02}\left(\left[L_{k}\right]\right)_{T}$ at $b^{\prime}:$ Spf $O_{K} \rightarrow T$. By using Theorem 3.1 the locus where $\left[L_{k}\right]$ is in the image of $\operatorname{sp}_{\bar{t}, \bar{s}}$ is the vanishing locus of $\gamma_{02}\left(\left[L_{k}\right]\right)$. By using this fact and the finitely generatedness of Néron-Severi groups, the Picard number jumping locus on a polydisk neighborhood $U$ (see [MP, Definition 4.1] for the definition) is written in the form of

$$
\bigcup_{\lambda \in \Lambda, \lambda \neq 0}(\text { zeros of } \lambda \text { in } U),
$$

where $\Lambda$ is a finitely generated $\mathbb{Z}$-submodule of (convergent power series ring on $U)^{n}$ (see [MP, Lemma 4.2]). Finally, by using linear algebraic arguments, they showed the above union is nowhere dense (see [MP, Proposition 5.1]).

\section{Acknowledgements}

The original manuscript of this paper (Section 1-4) was a letter to Professor Akio Tamagawa, in which the author answered to his questions. It was Professor Akio Tamagawa who pointed out a possibility of a generalization of the Maulik-Poonen results. The author thanks him for introducing these problems to him. He also thanks Professor Poonen for pointing out some English typos.

Finally, he sincerely thanks TOYOTA Central R\&D Labs. Inc. for offering him a special position in which he can concentrate on pure math research. The head of the company told me that if they would force me to do something else, then it would be against the policy of the company, and it would be against the philosophy of the founder of the company as well. The author also sincerely thanks Sakichi Toyoda for his philosophy, and the exectives for inheriting it from him for 80 years after his death.

\section{References}

[BO] Berthelot, P.; Ogus, A. F-Isocrystals and De Rham Cohomology. I Invent. Math. 72,(1983), 159-199.

[HK] Hyodo, O.; Kato, K. Semi-stable reduction and crystalline cohomology with logarithmic poles. Periodes $p$-adiques (Bures-sur-Yvette, 1988). Astérisque 223, (1994), 221-268.

[K1] Kato, K. Logarithmic structures of Fontaine-Illusie. Algebraic analysis, geometry, and number theory. Johns Hopkins University Press, Baltimore (1989), 191-224

[K2] Kato, K. Semi-stable reduction and p-adic étale cohomology. Periodes p-adiques (Bures-surYvette, 1988). Astérisque No. 223 (1994), 269-293.

[MP] Maulik, D., Poonen, B. Néron-Severi groups under specialization. preprint,

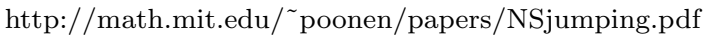

[N] Nakayama, C. Nearby cycles for log smooth families. Compositio Math. 112 (1998), no. 1, $45-75$.

[O] Ogus, A. F-Isocrystals and De Rham Cohomology. II Duke Math. J. 51 No. 4 (1984), 765-850. 
[S] Shiho, A. Crystalline fundamental groups II - log convergent cohomology and rigid cohomology. J. Math. Sci. Univ. Tokyo 9 (2002), 1-163.

[Tsu] Tsuji, T. p-adic étale cohomology and crystalline cohomology in the semistable reduction case. Invent. Math. 137,(1999), 233-411.

TOYOta CEnTRal R\&D LABS., INC. 41-1, Aza Yokomichi, Oaza Nagakute, NagakuteCHO, Aichi-Gun, Aichi-Ken, 480-1192, JAPAN

E-mail address: gokun@kurims.kyoto-u.ac.jp

E-mail address: gokun@mosk.tytlabs.co.jp 